Anna Palau, Victor Rouco, Roberto F. Luccas, Xavier Obradors, and Teresa Puig

\title{
6 Vortex dynamics in nanofabricated chemical solution deposition high-temperature superconducting films
}

\begin{abstract}
Artificial manipulation and control of vortex dynamics in $\mathrm{YBa}_{2} \mathrm{Cu}_{3} \mathrm{O}_{7}$ (YBCO) films have always been a complex issue, especially since high thermal excitations but also strong vortex pinning capabilities coexist in this material. Thus, artificial nanofabrication strategies able to generate competing effects with intrinsic microstructural defects need to be achieved. Lithography tools are widely used to create model systems with controlled pinning potentials in superconductors. However, these techniques easily disturb the optimal oxygen film doping in YBCO films and consequently the overall performances degrades.

We have optimized the use of two different high-resolution nanolithography approaches, Focused Ion Beam Milling and Electron Beam Lithography, to artificially and locally modify the pinning landscape of YBCO films grown by chemical solution deposition (CSD). Three different nanofabricated systems will be discussed, which resulted in ideal structures to manipulate vortex dynamics in CSD-YBCO thin films with strong intrinsic pinning centers. In particular, we observed artificial granularity effects, nanowall pinning, and positive and negative rectification effects. We will report on our understanding of all these effects and potential expectations.
\end{abstract}

\subsection{Introduction}

$\mathrm{YBa}_{2} \mathrm{Cu}_{3} \mathrm{O}_{7-\mathrm{d}}(\mathrm{YBCO})$ is ${ }^{4}$ the technologically most relevant high-temperature superconductor, highly explored for practical applications [1-6]. The achievement of artificial pinning centers (APC) in YBCO films is of major concern when pinning forces need to be optimized, in coated conductors (long-length epitaxial YBCO films on buffered flexible metallic substrates), or when they need to be exploited for electronic applications, requiring flux quanta manipulation. However, flux pinning in cuprate superconductors requires control of the defect structure on a nanometric scale and this is a cumbersome problem. The primary concern is to develop efficient techniques for

4 Current address for RF Luccas: Instituto de Física Rosario (IFIR, CONICET), Bv. 27 de Febrero 210bis, S2000EZP Rosario, Argentina.

Anna Palau, Victor Rouco, Roberto F. Luccas, Xavier Obradors, Teresa Puig, Institut de Ciència de Materials de Barcelona, CSIC, Campus UAB, 08193 Bellaterra, Spain 
tailoring the intrinsic microstructure of the material, which will strongly depend on the growth technique. Different routes have been used towards the preparation of high-quality YBCO films and coated conductors, based on both physical and chemical methodologies [7-14]. The Chemical Solution Deposition (CSD) techniques appear as an alternative to expensive vacuum-based physical technologies and targets nextgeneration YBCO film applications. This chapter will focus on the progress achieved in the nanofabrication of artificial pinning centers in YBCO films grown by CSD.

\subsection{Chemical solution deposition (CSD)}

Chemical Solution Deposition (CSD) technology has become one of the most appealing alternatives towards affordable production of long-length high-temperature superconducting tapes because it is a flexible, scalable and low-cost process. The CSD approach has been applied to the preparation of thin films of functional oxides in many different fields $[15,16]$, however its use to obtain epitaxial films has been less explored [17]. The field of HTS superconductors has been the driving force in this case [18]. The most cumbersome issue to achieve high-performance coated conductors (CC) has been to find a suitable methodology to grow epitaxial YBCO thin films and nanocomposites based on CSD on flexible metal substrates, and here, the greatest progress has been made based on the use of metal-trifluoroacetates (M-TFA) as metal-organic precursors [1922]. The main advantage of these precursors is that they decompose in intermediate nanometric $\mathrm{Cu}$ and $\mathrm{Y}$ oxides and $\mathrm{BaF}_{2}$ phases, thus avoiding the formation of $\mathrm{BaCO}_{3}$ whose decomposition is cumbersome [23]. Chemical Solution Deposition is based on four processing steps schematically shown in Figure 6.1 and described in the following. For an extended version see [18, 20, 21].

\subsubsection{Precursor solution}

The first step is the preparation of the precursor solution using metal-organic precursors (mostly metal-carboxilate salts) containing the required cations to form the desired compound (Y, $\mathrm{Ba}$, and $\mathrm{Cu}$ for the case of $\mathrm{YBCO}$ ). In the pure TFA route, triflu-

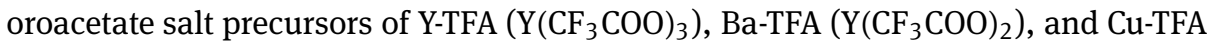
$\left(\mathrm{Cu}\left(\mathrm{CF}_{3} \mathrm{COO}\right)_{2}\right)$ are mixed in a stoichiometric ratio and dissolved in adequate solvents (methanol in most cases). The solubility, stability, and homogeneity of the solution will mainly depend on the specific salt, the metal ion concentration, the solvents, and possible additives used [18, 24].

The rheological parameters of the final precursor solution (concentration, viscosity, drying rate, surface tension, wettability, etc.) must be adapted to the selected deposition technique, allowing one to achieve a close control of the film thickness and final properties of the deposited film. 
(I) Precursor Solution

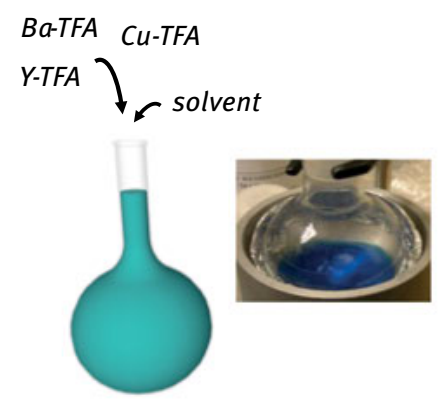

(II) Pyrolysis

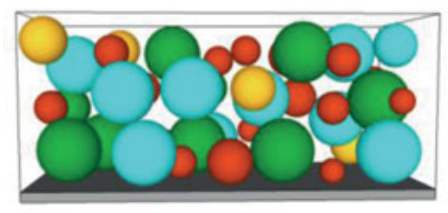

(II) Solution Deposition

(a)
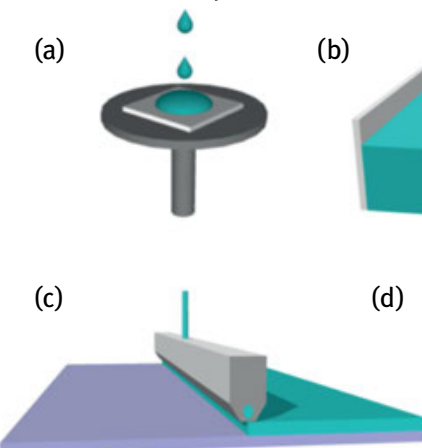

(d)

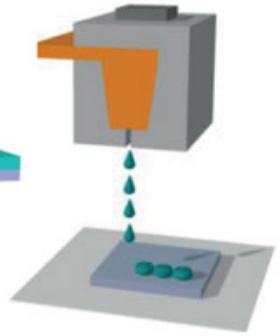

(IV) Growth and oxygenation

(b)
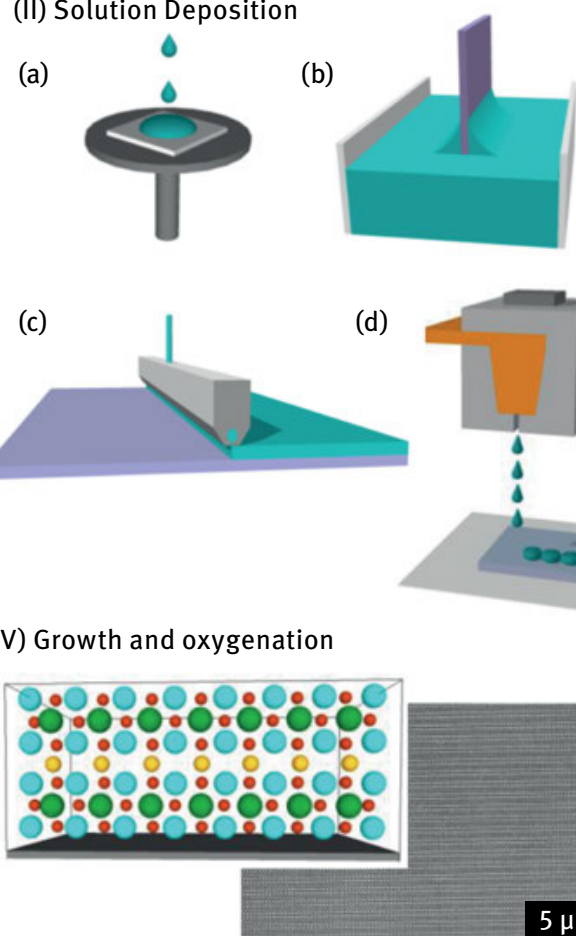

$5 \mu \mathrm{m}$

Fig. 6.1: Schematic representation of the four processing steps for Chemical Solution Deposition (CSD) growth of YBCO. (I) Precursor solution preparation. (II) Solution deposition by (a) spin coating, (b) dip coating, (c) slot-die coating, and (d) ink-jet printing. (III) Pyrolysis to obtain amorphous and nanocrystalline intermediate phases. (IV) Growth and oxygenation to achieve highly epitaxial films.

\subsubsection{Solution deposition}

The second step is the deposition of the coating solution onto the substrate. This is mainly performed by spin coating at laboratory scale, or dip coating, slot-die coating, and ink-jet printing, at industrial scale.

Spin-coating is a widespread deposition technique that consists of dropping a small amount of solution onto the center of a rotating substrate. The film thickness and homogeneity, mainly depends on the spinning parameters: rotation speed, acceleration, time, and also on more complex parameters such as environmental humidity and substrate temperature.

Dip coating is based on the immersion of the substrate in a liquid bath and the formation of a liquid film on withdrawal of the substrate from the coating fluid. The main parameters in the dip-coating process are the immersion, the dwell time that the substrate remains fully immersed and motionless, and the withdrawal speed. The faster the film is removed from the bath, the thicker is the final coating. 
In the slot-die coating process a precise measure of liquid is supplied onto the substrate and dispersed at a controlled rate while a coating die is precisely moved relative to the substrate. The wet film quality and thickness is controlled by the flow rate, coating width, and speed.

In the case of ink-jet printing, the concept for film deposition involves the precise positioning of very small volumes of fluid (1-100 picoliters) on a substrate. In this case understanding of the main physical processes that operate during ink-jet printing: generation of droplets, positioning, interaction of droplets with the substrate, and drying is key to achieving the desired deposited films [25, 26].

\subsubsection{Pyrolysis}

After a film drying process, the organic matter of the deposited films is decomposed. This is called the pyrolysis process. Typically, this involves thermal treatments up to $300-400^{\circ} \mathrm{C}$, typically in humid oxidizing atmospheres, in standard tubular furnaces. Water vapor in the gas flow prevents Cu-TFA sublimation via hydrostabilization [18].

The pyrolysis time, temperature, heating rates, and oxygen partial pressure must be optimized according to the kinetics of the precursor decomposition.

The main difficulty in achieving homogeneous pyrolyzed films arises from the strong film shrinkage occurring during the pyrolysis process. In this process, the stress relief must be slow enough to avoid film inhomogeneities such as buckling or macrocracks. In the particular case of the TFA route the pyrolysis reaction is:

$$
\begin{aligned}
& \mathrm{Y}\left(\mathrm{CF}_{3} \mathrm{COO}\right)_{3}+2 \mathrm{Ba}\left(\mathrm{CF}_{3} \mathrm{COO}\right)_{2}+3 \mathrm{Cu}\left(\mathrm{CF}_{3} \mathrm{COO}\right)_{2}+\mathrm{H}_{2} \mathrm{O}+\mathrm{O}_{2} \\
& \rightarrow \mathrm{CuO}+\mathrm{Ba}_{2-\chi} \mathrm{Y}_{\chi} \mathrm{F}_{2+\chi}+\mathrm{Y}_{2} \mathrm{O}_{3}+\text { volatile phases } .
\end{aligned}
$$

The pyrolysis process leaves the film as a mixture of amorphous and nanocrystalline intermediate phases, which should maintain homogeneity and integrity at the nanoscale.

\subsubsection{Growth and oxygenation}

The final step in the CSD TFA-route process is the crystallization of the film into the desired oxide phase following the reaction:

$$
\frac{3}{2 \mathrm{CuO}}+\mathrm{Ba}_{2-x} \mathrm{Y}_{x} \mathrm{~F}_{2+y}+\frac{1}{4 \mathrm{Y}_{2} \mathrm{O}_{3}}+y \mathrm{H}_{2} \mathrm{O} \rightarrow \frac{1}{2 \mathrm{YBa}_{2} \mathrm{Cu}_{3} \mathrm{O}_{7-\delta}}+2 y \mathrm{HF} .
$$

In this step the pyrolyzed film is heated at high temperature $\left(T \sim 700-800^{\circ} \mathrm{C}\right)$, and the nucleation, growth, and densification of the YBCO layer is produced in a humid atmosphere-controlled tubular furnace to decompose the fluorinated compounds 
and enable YBCO formation. CSD-YBCO films grow following a Volmer-Weber 3D island mode where initial nuclei are stabilized, then they coarsen, coalescence, and finally grain boundaries need to be healed to obtain a dense film. For the control of these nucleation and growth processes of the YBCO film, a proper selection of the oxygen partial pressure $\left(\mathrm{PO}_{2}\right)$-temperature region of the phase diagram is mandatory, but also water partial pressure, gas flow, and heating rate are very relevant parameters influencing both processes in different manners [18]. After the adjustment of all these parameters, epitaxial YBCO films with a $c$-axis perpendicular to the substrate plane are obtained. The growth conditions are highly dependent on the substrate surface quality and the lattice mismatch and therefore they must be adjusted for each coated conductor architecture [6]. After growth, the film must be oxygenated to reach the final superconducting orthorhombic structure. Once optimal growth and oxygenation conditions are attained, epitaxial thin films of very high quality can be produced with $J_{c}=3-5 \mathrm{MA} / \mathrm{cm}^{2}$ at self-field and $77 \mathrm{~K}$ in thicknesses of $200-500 \mathrm{~nm}$. Larger film thicknesses needed to achieve higher critical currents are mostly achieved through the use of multideposited layers [27].

The ultimate superconducting performance of the YBCO films will strongly depend on the natural nanoscale defect structure formed during CSD processing [28]. It is well known that natural defects, such as dislocations, vacancies, intergrowths, or twin boundaries (TBs) can act as effective pinning centers and are the source of high critical currents in YBCO films [18, 29].

\subsection{Artificial pinning centers in CSD-YBCO films}

Tailoring the vortex pinning landscape in YBCO films is presently one of the major challenges because of its relevance to applications requiring manipulation of flux quanta or enhanced critical currents. However, artificial control of the pinning scenario in YBCO films is a complex issue, especially because of their high thermal excitations and their already strong intrinsic pinning capabilities due to the presence of many natural defects. Thus, artificial nanofabrication strategies able to generate competing pinning sites with an intrinsic nanoscale defect structure need to be used.

Different routes have been developed to introduce effective artificial pinning centers (APC) in CSD-YBCO films, going from scalable processes where randomly oriented second-phase nanoparticles have been spontaneously segregated into the YBCO matrix, to designed model systems with ordered nanostructures.

In the first approach, we modify the precursor solution by introducing specific amounts of particular metal-organic salts (Zr-, Hf-, Ta-carboxilates) which form nanocomposite films with secondary nanophases $\left(\mathrm{BaZrO}_{3}, \mathrm{BaHfO}_{3}, \mathrm{Ba}_{2} \mathrm{YTaO}_{6}\right)$ spontaneously segregated within the YBCO matrix during growth. It has been demonstrated that the presence of these randomly oriented nanoparticles induces the formation of a large density of stacking faults that produce strong lattice distortions in 

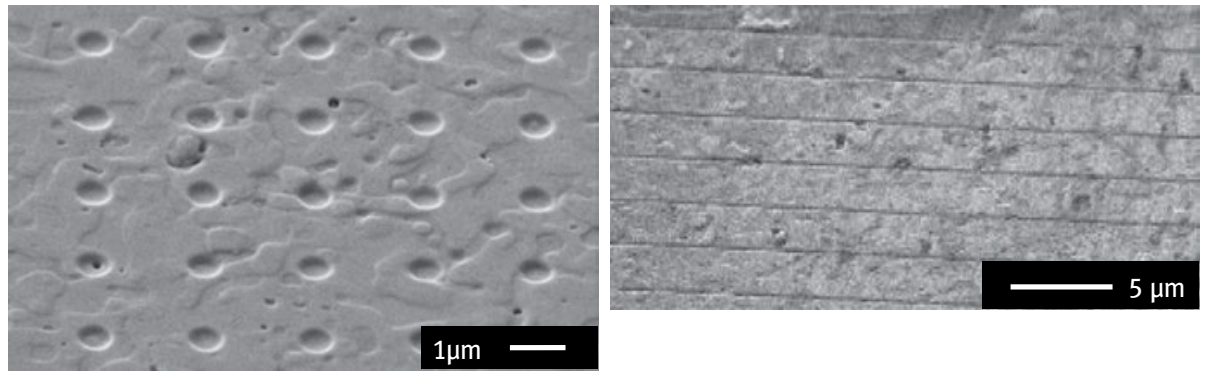

(a)

(b)
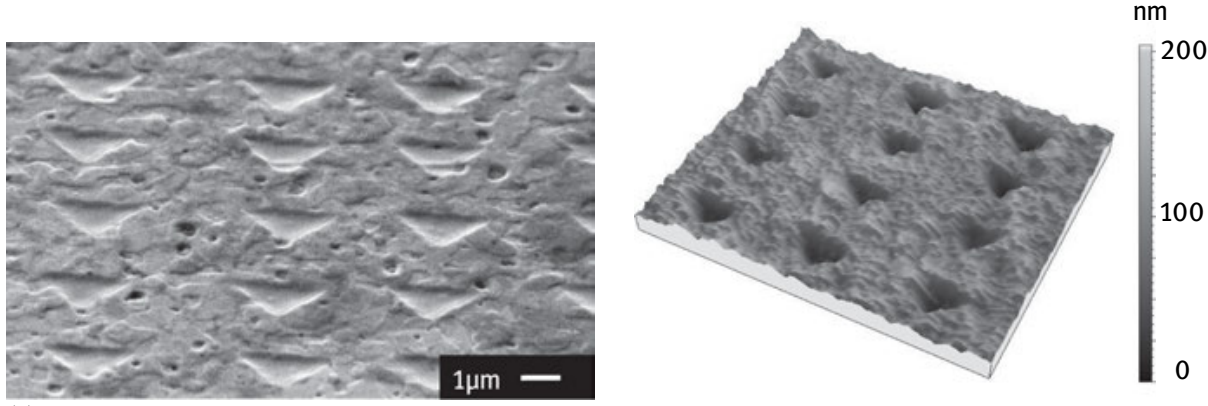

(c)

(d)

Fig. 6.2: SEM pictures of several CSD-YBCO bridges patterned with different structures. (a) Circular antidots, (b) blind trenches, (c) triangular antidots. (d) AFM image of the pattern with triangular antidots shown in (c).

the YBCO matrix, which act as very efficient strong isotropic pinning centers [10, 30]. YBCO films with $\mathrm{BaZrO}_{3}$ (BZO) nanoparticles, for example, have shown enhanced pinning forces ( $75 \mathrm{GN} / \mathrm{m}^{2}$ and $600 \mathrm{GN} / \mathrm{m}^{2}$ at $3 \mathrm{~T}, 65 \mathrm{~K}$ and $9 \mathrm{~T}, 10 \mathrm{~K}$, respectively) [4], more than five times larger than standard films grown by the same process [10]. Recently, a more advanced strategy where preformed nanoparticles are stabilized in the YBCO precursor solutions, from which the nanocomposite films are grown, has also been developed [31]. The idea behind this is to have greater control of nanoparticles size.

These types of artificial defects are distributed arbitrarily in the superconducting nanocomposite film and understanding vortex physics in these systems is always more complicated. A different strategy can be followed, based on designed model systems with ordered nanostructures that enable one to properly engineer the pinning landscape, where vortex dynamic behavior can be better controlled and manipulated [32].

In this chapter we will report on this latter approach, aiming to study different model superconducting systems with APC. We will use different nanofabrication strategies, based on high-resolution lithography techniques, able to locally modify the superconducting material at nanometric scale, inducing pinning sites with controlled parameters (distribution, density, shape, and size). Antidots completely perforating 
the YBCO layer, blind antidots and trenches are the different nanostructures that we have fabricated, by using Focused Ion Beam (FIB) milling and Electron Beam Lithography (EBL). This has resulted in several new physical phenomena that will be discussed in the following section. Figure 6.2 show several examples of CSD-YBCO films patterned with these APCs.

\subsubsection{Electron beam lithography}

Electron Beam Lithography (EBL) consists of the electron irradiation of a surface that is covered with a resist sensitive to electrons. This high-resolution lithographic process is able to create submicronic structures in the polymeric layer irradiated with a focused electron beam. Figure 6.3 shows the three different steps required to pattern a film by EBL: Exposure of the electron sensitive material, development of the resist, and pattern transfer to the film. Each individual step, with a great number of parameters that must be optimized, contribute to the final resolution of the pattern.

The first step starts with the preparation of a resist layer on top of the film by spin coating. For positive polymer resists, usually polymethyl methacrylate (PMMA), the polymer irradiated area can be eliminated with a development process. The used polymer and spin parameters will determine the final resist thickness. After the deposition, the resist layer is soft-baked on a hot plate and the film is then ready for irradiation. It is important to remark that the exposed area cannot be observed once the resist is deposited so a good sample alignment is necessary before exposure.

The exposure procedure strongly determines the final resolution of the patterned structures. This process comprises several points: pattern design and sample alignment, optimization of the electron beam parameters (working distance, spot size, voltage, focus), calibration of the write field (working area that will be exposed), dose (amount of electrons per unit area that the resist receives), and beam speed.

The right dose value to obtain good patterned features in the resist layer depends on the resist sensitivity, thickness, and development conditions. The development process consists of sample immersion in a developer solution to remove the irrigated
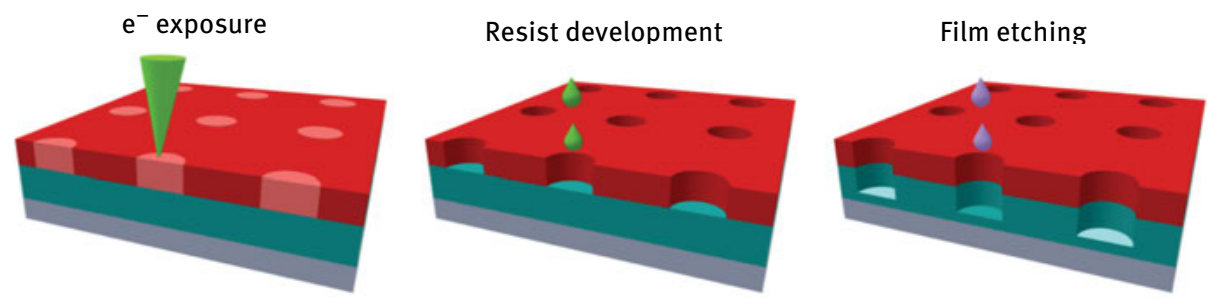

Fig. 6.3: Schematic representation of the different steps in the Electron Beam Lithography process. 
resist and obtain the desired pattern. This process must also be optimized to avoid under- or over-developed features.

Finally, the pattern is transferred to the film by means of wet or dry etching, using the resist as a mask. In this process the etching parameters are critical to obtaining a fine reproduction of the desired structures on the film. The etching depth can be tuned to transfer the pattern to the whole film thickness or to just mill a part of the film.

The different studies presented in this work were performed with a field emission SEM QUANTA FEI 200 FEG-ESEM. In all cases we deposited a $100 \mathrm{~nm}$ thick positive PMMA resist, prebaked at $180^{\circ} \mathrm{C}$ over $60 \mathrm{~s}$. For the irradiation we changed the voltage from $10 \mathrm{kV}$ to $30 \mathrm{kV}$ and adjusted all beam parameters for the best patterning conditions. The YBCO films were wet etched with a diluted solution of orthophosphoric acid.

\subsubsection{Focused ion beam lithography}

In the case of Focused Ion Beam (FIB) lithography, the irradiating ions are heavy enough to directly etch the YBCO film, without the need of any mask. With this technique, Gallium ions are focused and accelerated to the sample surface, by using electrostatic lenses and coils. The high energy of these ions produces direct milling of the material at the nanoscale (Figure 6.4).

Gallium is currently the most commonly used ion source for FIB instruments since it is metallic, has a low melting temperature, low volatility, and low vapor pressure, offering excellent mechanical, electrical and vacuum properties. Moreover, the atom is large enough to mill heavy elements.

One of the main advantages of FIB lithography is that it enables direct processing at the nanometer scale with the possibility to image the sample while doing the irradiation. Dual-beam (FIB/SEM) systems offer the option to monitor the ion beam milling with SEM imaging, without damaging the sample. However, in direct milling, secondary effects occur around the irradiated areas, which could modify film properties. Effects can include implantation of $\mathrm{Ga}^{+}$ions in the YBCO matrix, redeposition of small amounts of sputtered material, or sample amorphization (see Figure 6.4). The secondary effects can be minimized by reducing the milling rate.

An accurate optimization of the FIB milling parameters has to be performed in order to obtain high-resolution patterns, whilst minimizing sample damage during the milling process. The most relevant factors are beam voltage, current, dose (number of ions per unit area), and milling rate.

In this work we used a Carl Zeiss Crossbeam 1560 XB system, with an SEM and a FIB column, combining the applications of the focused ion beam with imaging performance. We used a constant beam voltage of $30 \mathrm{kV}$ with variable beam currents (between 5-200 pA), depending on the milling rate. 


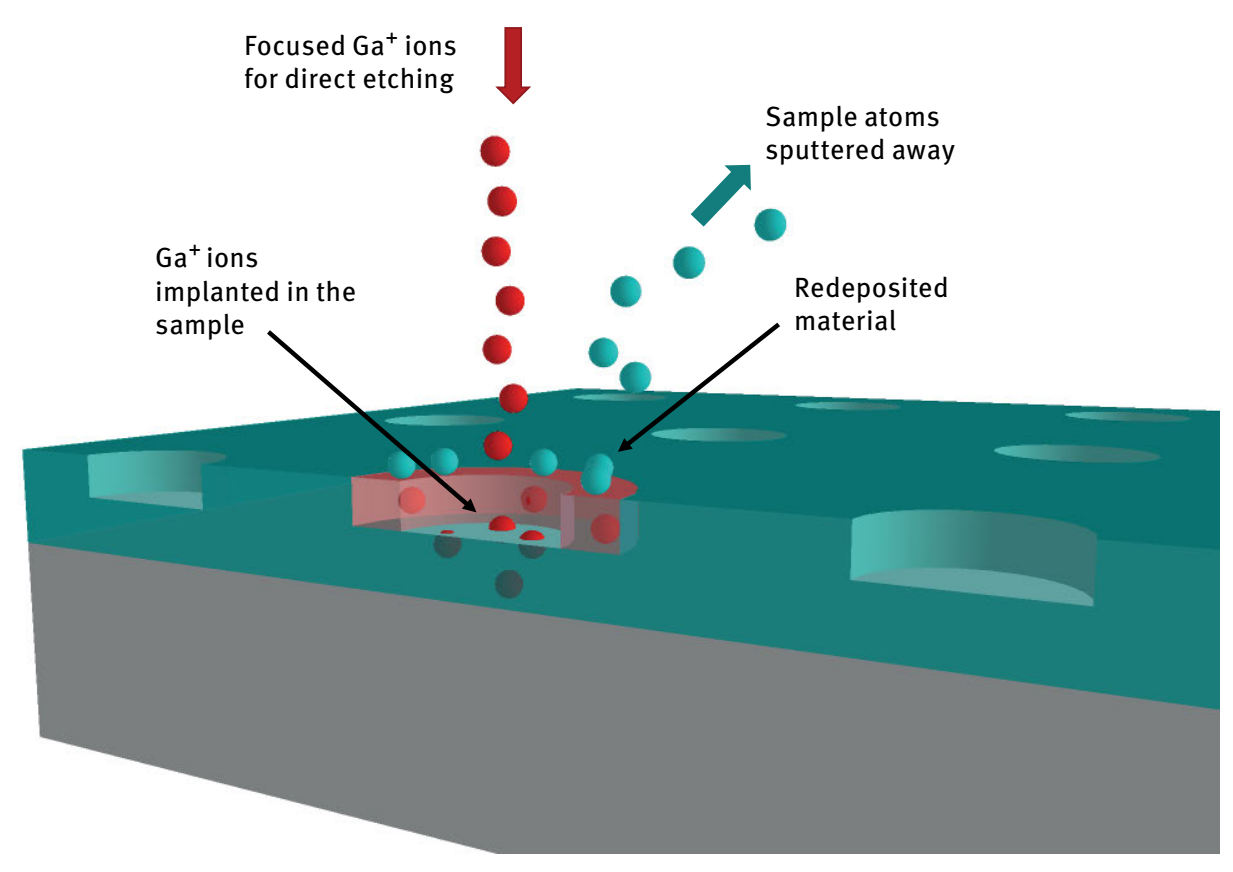

Fig. 6.4: Schematic representation of Focused Ion Beam etching.

\subsection{Manipulating vortex dynamics in YBCO films with APC}

\subsubsection{Physical characterization techniques}

The aim of this section is to elucidate the effect of different artificial pinning centers, generated by EBL and FIB, on the vortex lattice in CSD-YBCO thin films. This is achieved through measurements of the transport critical current density with temperature and magnetic field. Moreover, the interaction vortex-defect will be explored by means of magnetic decoration at low magnetic fields.

\section{Transport measurements}

Transport measurements, where current is applied through a superconducting sample while the voltage across it is measured, are a direct and reliable way to study vortex pinning and dynamics. With this technique we are able to define current tracks in the desired positions of the YBCO film, which combined with the nanofabricated APC techniques, allow us to analyze and compare the effect of different pinning centers in the same sample. All measurements presented here were performed in a Quantum Design Physical Properties Measurement System (PPMS) using a four-point bridge configuration with silver contacts with a contact resistance lower than $10^{-6} \Omega \mathrm{cm}^{2}$. Mea- 


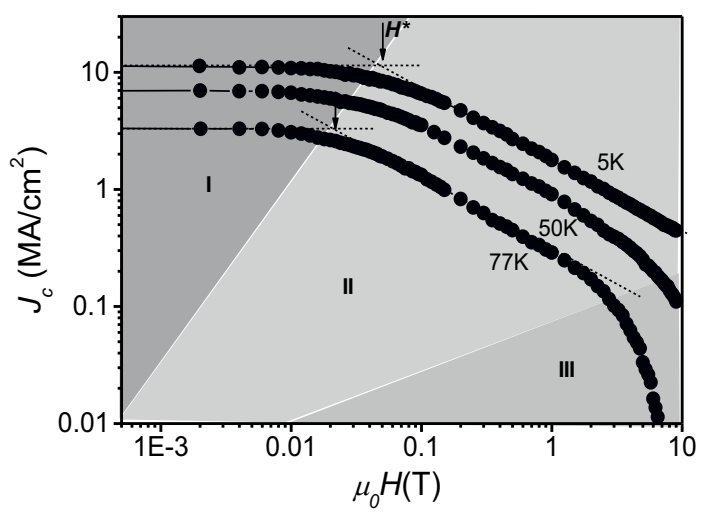

Fig. 6.5: Magnetic field dependence of $J_{c}$ at temperatures shown for a standard YBCO film. Different colors indicate regions determined by (I) single vortex pinning, (II) collective vortex pinning, (III) irreversibility line approach. Arrows indicate the characteristic field $H^{*}$.

surements were performed over a range of temperatures from 5 to $77 \mathrm{~K}$ and magnetic fields of up to $9 \mathrm{~T}$ applied perpendicular to the $c$-axis.

Figure 6.5 shows the magnetic field dependence of the critical current density, $J_{\mathrm{c}}(H)$, obtained for a standard CSD-YBCO thin film at different temperatures. Notice that it is possible to distinguish three different regimes in the $J_{\mathrm{C}}(H) \log$-log diagram [33, 34]. The first regime is at low magnetic field and corresponds to the single vortex pinning regime, where each vortex is individually pinned to a defect, thus $J_{\mathrm{c}}$ is fieldindependent. In the intermediate regime, above the characteristic field $H^{*}$, the density of vortices becomes greater than the density of defects and interactions between them become important. This region is described by collective vortex pinning interactions that are described by a power law $J_{\mathrm{c}} \sim H^{-\alpha}$, where $\alpha$ depends on the type of interaction. In the third regime at high fields, $J_{c}$ decays strongly since it approaches the irreversibility line. Thus, depending on the applied magnetic field and temperature, different vortex motion regimes can be studied and the corresponding effects of different APC can be analyzed.

\section{Bitter decoration}

Bitter decoration is a visualization technique that allows one to provide a direct illustration of the vortex lattice in a superconductor. With this procedure we are able to study the interaction of vortices with the nanostructures defined in the YBCO films.

A Bitter decoration experiment of a superconducting specimen consists of the evaporation of tiny ferromagnetic clusters on the sample surface when this is in the superconducting state, so that the ferromagnetic clusters get attracted to the vortex positions. The sample is placed in an evaporation chamber with controlled pressure, temperature, and magnetic field. The critical conditions for optimal deposition are 
defined by the amount of ferromagnetic material evaporated, the source-to-specimen distance, the helium vapor pressure (which strongly defines the size of the ferromagnetic clusters), and the magnetic field modulation within the sample. In order to ensure large field modulations in the sample, the applied magnetic field must be rather small $[35,36]$.

After decoration, the sample is warmed up and the vortex positions, marked by the attracted ferromagnetic clusters, are imaged by SEM. One of the main advantages of this technique is that it allows the observation of vortices in large areas in the same experiment and thus, interaction between vortices and defects located at different regions of the sample can be studied.

A standard approach used to characterize ordering of the vortex lattice distribution from a decorated image is the so-called Delaunay triangulation. For a set of points (vortex positions) in 2D, a Delaunay triangulation of these points ensures the circumcircle associated with each triangle contains no other point in its interior. By using this triangulation, each vortex site is connected to its nearest neighbors by segments, thus providing information of vortex coordination. Moreover, additional information to quantify the lattice ordering is provided by the vortex density $(\rho)$ autocorrelation function, which gives the crosscorrelation of the $2 \mathrm{D}$ vortex distribution with itself, as a function of small displacements from the original position [37].

$$
G(\boldsymbol{r})=\langle\rho(\boldsymbol{R}) \rho(\boldsymbol{R}+\boldsymbol{r})\rangle_{\boldsymbol{R}} .
$$

This function presents an absolute maximum in the center of the image (perfect autocorrelation) and indicates other maxima when by image displacement other vortices overlap the original vortex positions. For vortex lattices with specific symmetry, this study clearly identifies the mentioned symmetry.

In this work, we used a homemade decoration system $[37,38]$ with an Fe filament, in a chamber with variable He pressure (down to 30 mtorr), a fixed temperature of $\sim 4.2 \mathrm{~K}$, and a controllable external field (from 0 to $10 \mathrm{mT}$ ) to study different systems.

Figure 6.6a and b show an SEM image of a YBCO single crystal, grown with very few defects, decorated at $1.6 \mathrm{mT}$ and $6.6 \mathrm{mT}$, respectively, and the associated Delaunay triangulations and autocorrelation functions. Green and red points in the triangulation correspond to vortex coordination equal or different to six, respectively.

At low magnetic field values $(1.6 \mathrm{mT})$, the sample remains at the single vortex pinning regime (Region I in Figure 6.5), in which vortex-defect interaction prevails over vortex-vortex interaction and thus a disordered vortex lattice, without any detected symmetry, is obtained. At higher applied fields (6.6 mT), vortex dynamics are dominated by collective pinning effects and a great majority of regions with six-fold orientation order are observed. The autocorrelation function in this case indicates a clear hexagonal symmetry, characteristic of the ordered Abrikosov lattice.

A completely different situation arises, if the decoration is performed in a CSDYBCO thin film with a large amount of intrinsic defects. In this case, no ordering or symmetry at all is observed even at the highest magnetic field values measured (Fig- 

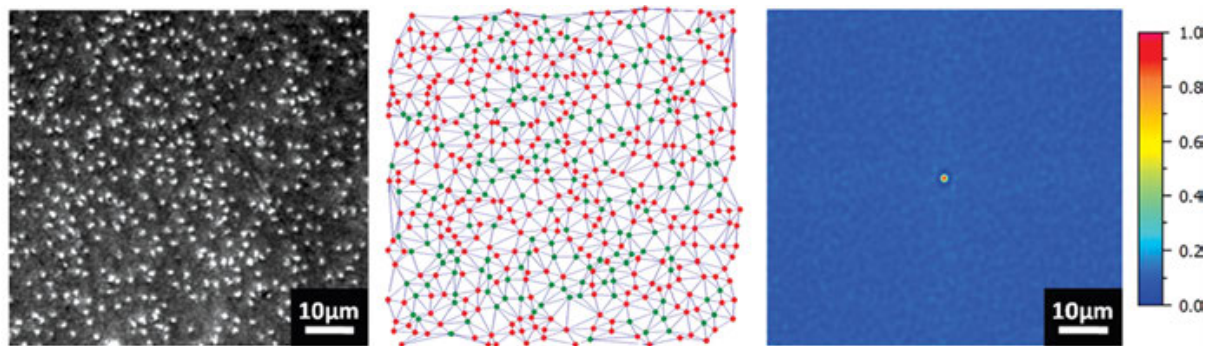

(a)
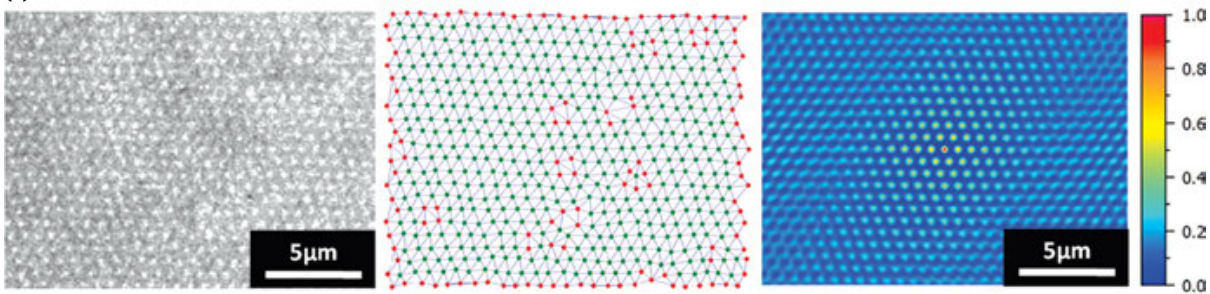

(b)

Fig. 6.6: SEM images showing the Bitter decoration of a YBCO single crystal at (a) $1.6 \mathrm{mT}$ and (b) $6.6 \mathrm{mT}$ at $4.2 \mathrm{~K}$ with the associated Delaunay triangulation and autocorrelation function. White spots in the SEM image mark the attracted ferromagnetic clusters.

ure 6.7), since for this range of fields the system stays in the single vortex pinning regime due to the strong vortex-defect interaction.

Thus, very efficient artificial pinning sites must be introduced in these films to overcome the already existing natural defects if one wants to study the modifications of vortex dynamics by artificially modeled pinning potentials.
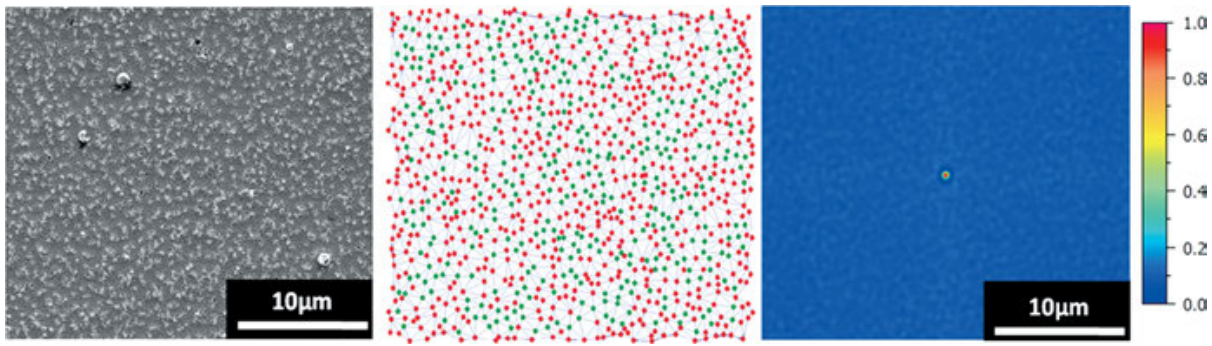

Fig. 6.7: SEM images showing the Bitter decoration of a CSD-YBCO thin film at $5 \mathrm{mT}$ and $4.2 \mathrm{~K}$ with the associated Delaunay triangulation and autocorrelation function. White spots in the SEM image mark the attracted ferromagnetic clusters. 


\subsubsection{Artificially ordered pinning center arrays}

In the following section, we will discuss several nanofabricated systems, prepared by both electron beam lithography and focused ion beam lithography, and some physical phenomena that we have gathered from them.

\section{YBCO films with nanodots}

The first studied system is a CSD-YBCO film patterned with circular antidots completely perforating the whole film thickness. Different squared lattices of antidots with a period of $2 \mu \mathrm{m}$ were obtained by FIB lithography. Figure 6.8 shows SEM pictures of two YBCO films patterned with lattices of different antidot diameter $(0.2$ and $0.5 \mu \mathrm{m})$.

Transport critical current versus magnetic field $\left(J_{c}\right.$ versus $H$ ) measurements were performed in order to determine the effect of the two antidot arrays in the different vortex pinning regimes described in Figure 6.5. The $J_{\mathrm{c}}(H)$ curves measured at $77 \mathrm{~K}$ for a reference sample and the two bridges patterned with antidots are shown in Figure 6.9a. The first observation is that in all cases the self-field critical current density, $J_{\mathrm{c}}^{\mathrm{sf}}$, calculated with the full bridge cross section, is reduced with the antidot patterning. This decrease in $J_{\mathrm{c}}^{\mathrm{sf}}$ is much higher than that expected according to the reduction in the effective cross-sectional area, due to the presence of the antidots. The value of $J_{\mathrm{c}}^{\text {sf }}$ has been reduced $70 \%$ and $90 \%$ for the antidot array with a diameter of $0.2 \mu \mathrm{m}$ and $0.5 \mu \mathrm{m}$, respectively, while the reduction in cross section is $10 \%$ and $25 \%$, respectively. The value of $T_{\mathrm{c}}$ is much less affected with reductions of less than $5 \mathrm{~K}$ observed in all cases. Thus, during the antidot milling we are not damaging the complete YBCO layer but just producing an amorphization of the material in the area surrounding the antidots (see Figure 6.4), probably associated to an overmilling of the sample or to the

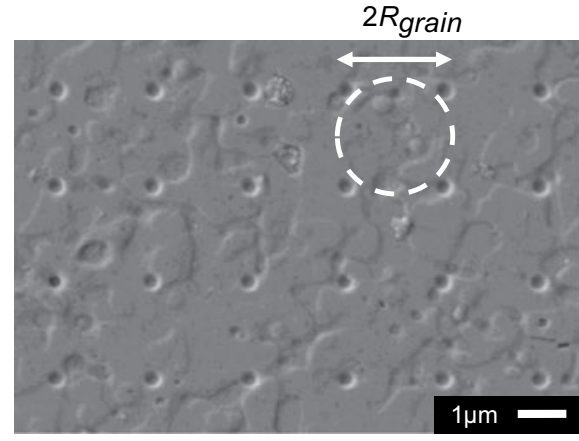

(a)

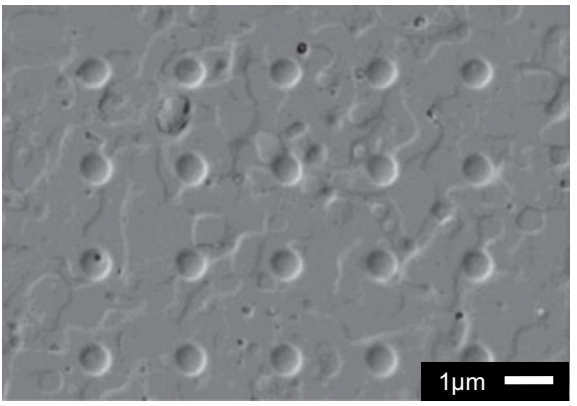

(b)

Fig. 6.8: SEM images of CSC-YBCO bridges with square patterns of circular antidots, completely perforating the film thickness, with a diameter of (a) $0.2 \mu \mathrm{m}$ and (b) $0.5 \mu \mathrm{m}$. Dashed lines show an effective grain determined by the antidot pattern. 

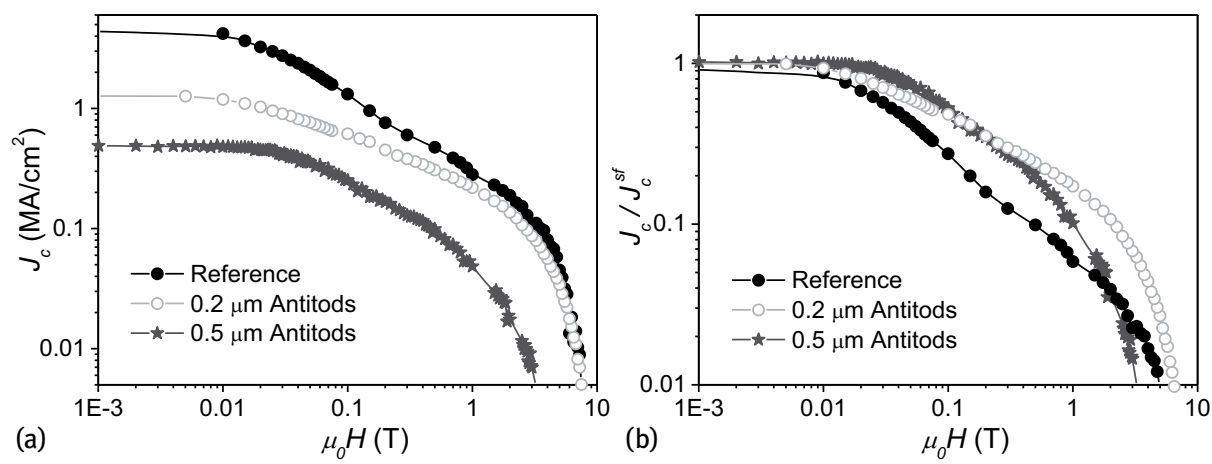

Fig. 6.9: (a) Magnetic field dependence of the critical current density measured at $77 \mathrm{~K}$ for a reference film and two bridges patterned with antidots. (b) $J_{c}(H)$ curves shown in (a) normalized to self-field $J_{c}$.

use of a too-high current beam ( $30 \mathrm{pA}$ in both cases). This exemplifies the care that needs to be taken with these types of nanofabrication technique.

Despite the observed reduction in self-field critical current density, the presence of the antidot lattice clearly modifies the magnetic field dependence of the sample. By comparing the $J_{\mathrm{c}}(H)$ curves, normalized to self-field, (Figure 6.9[b]) we observe that the presence of antidots changes the pinning landscape, producing a smoother $J_{c}$ magnetic field dependence in the patterned bridges. However, because of the large decrease in $J_{c}^{\text {sf }}$ no effective pinning enhancement is detected at any field. We will see in the following section that by strongly limiting the damage of the regions next to the antidots, $J_{c}^{s f}$ reduction is avoided and effective pinning enhancements are induced with this nanofabrication technique.

The most relevant characteristic observed in these systems is that the $J_{\mathrm{c}}(H)$ curves show a marked hysteresis depending on the direction of the field sweep. This is illustrated in Figure 6.10 where we have plotted the $J_{\mathrm{c}}$ field dependence measured by decreasing the magnetic field from $0.1 \mathrm{~T}$ to $-0.1 \mathrm{~T}$ and increasing it back to $0.1 \mathrm{~T}$ (see dashed arrows in the figure), for a track with antidots (Figure 6.10a) and a reference track (Figure 6.10[b]), at different temperatures.

While no hysteresis at all is observed for the reference track at any temperature, a substantial hysteretic behavior appears in the patterned track as the temperature is reduced. This behavior appears similar to that inductively measured in granular YBCOcoated conductors $[39,40]$ and artificial multigranular YBCO films [41], which are explained on the basis of reverse field components induced by the trapped current loops generated in inhomogeneous films. The patterned antidots, completely perforating the film thickness, emulate the current flow restricting behavior of grain boundaries thus producing a square network of artificial grains with an effective radius, $R_{\text {grain }}$, determined by the antidot distance (see Figure 6.8a). When performing the $J_{\mathrm{c}}(H)$ measurements shown in Figure 6.10a, return magnetic fields $\left(H_{\text {return }}\right)$ appear at the edges 

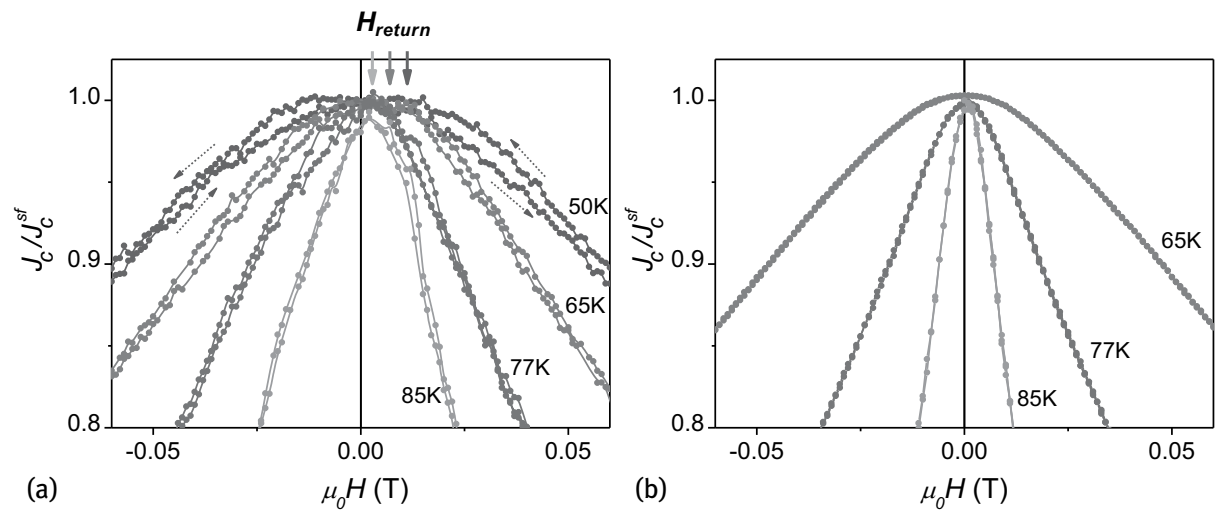

Fig. 6.10: Critical current density, normalized to self-field, versus applied magnetic field for (a) a CSD-YBCO bridge patterned with circular antidots and (b) a reference bridge, measured with a decreasing and increasing field at different temperatures. Dashed arrows show the field sweep direction and solid arrows show the calculated returned field at each temperature (see text for details).

of the artificial grains, coming from trapped current loops at each grain, which will reduce the local magnetic field within the film $\left(H_{\text {local }}=H-H_{\text {return }}\right)$. Hence, the maximum critical current density peak measured at $H_{\text {local }}=0$ appears shifted at $H \sim H_{\text {return }}$. We have evaluated the value of $H_{\text {return }}$ generated by the artificial grains patterned in the film shown in Figure 6.10(a), using the model described in [39, 40], and considering that two grains are contributing at each point of the current percolative path.

$$
H_{\text {return }}=2 J_{\mathrm{c}} x t \text {, }
$$

where $t$ is the sample thickness and $x$ is a dimensionless factor numerically calculated depending on the ratio $R_{\text {grain }} / t$. Solid arrows in Figure 6.10a show the values of $H_{\text {return }}$ obtained at $77 \mathrm{~K}, 65 \mathrm{~K}$, and $50 \mathrm{~K}$, which are in agreement with the peak shift observed for $J_{\mathrm{c}}(H)$ at each temperature.

Besides the use of these structures as a systematic approach to investigating granularity effects in YBCO thin films, they are nice systems to model engineered hightemperature hybrid materials, in which superconducting ferromagnetic interactions could be analyzed. Such studies are performed by filling the nanodots with magnetic cobalt rods [42].

\section{Nanowalls}

In light of the feasibility of antidot lattices to modify the pinning landscape of YBCO films, in this section we will investigate the capability of this strategy to effectively increase the critical current density, just perforating part of the total sample thickness, trying to avoid the strong $J_{\mathrm{c}}^{\text {sf }}$ reduction observed in the case of antidots fully perforating the layer (see Figure 6.11). 
To do so, we patterned YBCO films, by means of FIB, with blind trenches and antidots using a slow-enough milling rate to properly define the desired structures without damaging the surrounding area.

The aim is to tune the depth, shape, and dimension of the artificially milled nanostructures to tailor the pinning capabilities of the YBCO films, defining the limits of maximum reduced cross section leading to a total pinning enhancement. The current beam and the milling time used during the FIB irradiation are crucial parameters to ensure minimum, or nonexistent, damage to the film, thus allowing fine control of the milled cross section and the associated self-field $J_{\mathrm{c}}$ reduction. These two parameters have been properly optimized and in all cases we used beam currents of the order of 5-20 pA, thus lower than $30 \mathrm{pA}$, which was recognized as being too high in the previous section.

Figure 6.12a shows the ratio of $J_{\mathrm{c}}$, calculated considering the full sample cross section, before and after patterning, for samples with different nanostructures. The value of $S_{\text {red }}$ corresponds to the percentage of cross-sectional area etched with the patterning.

$$
S_{\text {red }}(\%)=100 \frac{S_{\text {antidot }}}{S_{\text {total }}},
$$

where $s_{\text {antidot }}$ is the maximum area of blind antidots perpendicular to the current flow (red area schematically shown in Figure 6.11) and stotal the cross-sectional area of the unpatterned bridge.

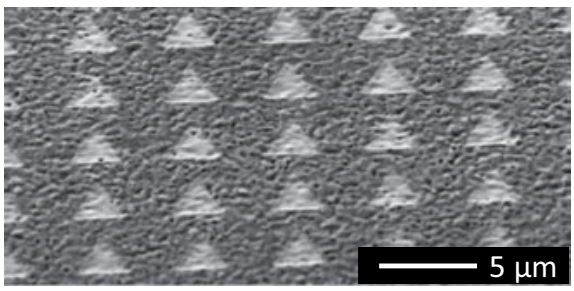

(a)

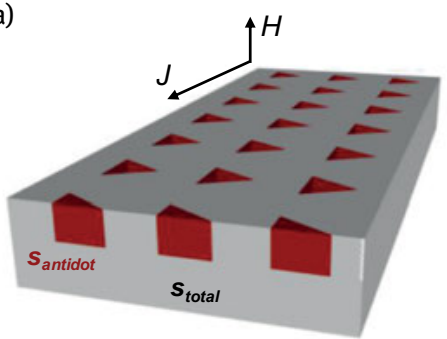

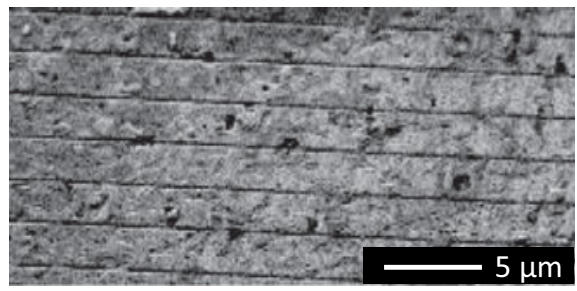

(b)

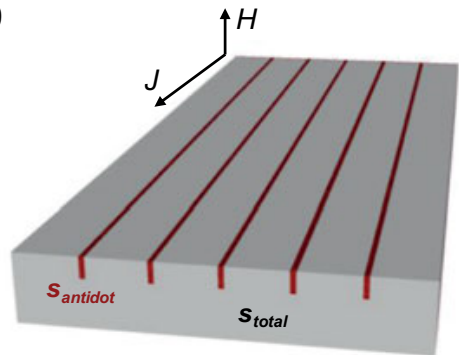

Fig. 6.11: SEM images of CSC-YBCO bridges patterned with blind (a) triangular antidots and (b) trenches. Schematic drawings show the cross section of patterned bridges. Red area corresponds to the area occupied by blind antidots (santidot) and stotal is the total cross section of the unpatterned bridge. 

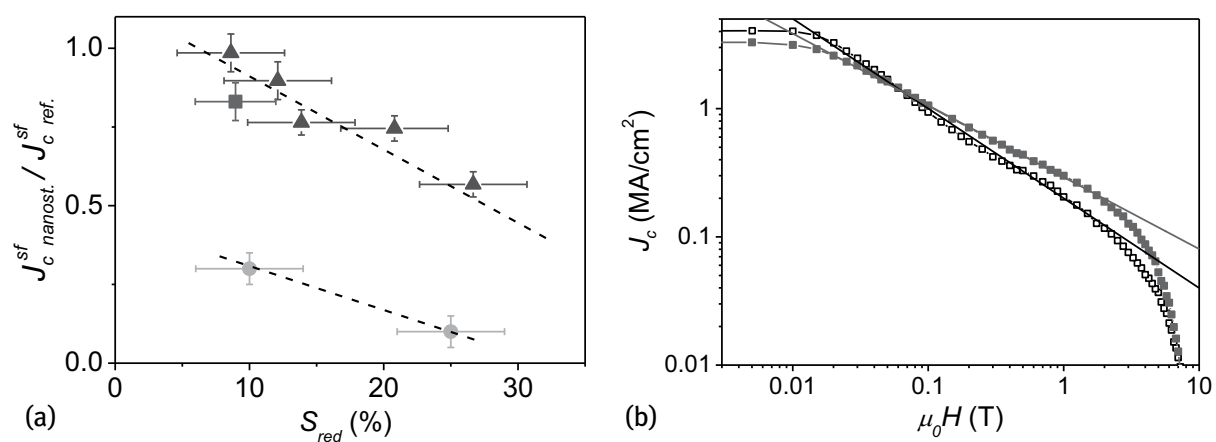

Fig. 6.12: (a) Ratio of self-field critical current density at $77 \mathrm{~K}$ after and before patterning as a function of Sred (defined in the text), for CSC-YBCO bridges with triangular blind antidots (triangles), trenches (square), and circular antidots fully perforating the film (circles). Dashed lines are a guide for the eyes. (b) $J_{c}$ versus magnetic field $H$ measured at $77 \mathrm{~K}$ for a pristine CSD-YBCO bridge (open symbols) and the same bridge nanostructured with longitudinal trenches (closed symbols). Lines are fits to the power law collective pinning region.

We have included in the graph the bridges patterned with triangular blind antidots (triangles), blind trenches (square), and the ones patterned with circular nanodots completely perforating the film thickness, described in the previous section (circles).

This data clearly evidences that the sample damage induced during milling is clearly diminished in the case of blind antidots which show a much smaller $J_{\mathrm{c}}$ reduction for similar $S_{\text {red }}$ values. A linear dependence between the $J_{\mathrm{c}}$ ratio and $S_{\text {red }}$ can be established in both cases although for samples patterned down to the substrate the self-field reduction is much higher. Figure 6.12b shows the $J_{\mathrm{c}}$ field dependence measured for a YBCO bridge with parallel blind trenches (shown in Figure 6.11b), before and after the patterning. As observed in the case of the system with small circular nanodots (open circles in Figure 6.9) the power law decay of $J_{c}$ is softened ( $\alpha$ is reduced) when we introduce the nanostructures. However, whereas in the case of the antidots $J_{\mathrm{c}}^{\mathrm{sf}}$ is strongly reduced after the milling $J_{\mathrm{c} \text { nanost. }}^{\mathrm{sf}} / J_{\mathrm{c} \text { ref. }}^{\mathrm{sf}}=0.30$, a much smaller decrease is generated with the blind trenches $J_{\mathrm{c} \text { nanost. }}^{\mathrm{sf}} / /_{\mathrm{c} \text { ref. }}^{\mathrm{sf}}=0.83$ which produces an effective enhancement of the pinning force at intermediate fields. Thus, the key point to maximize the pinning performance is determined by the balance of two opposing effects: (1) the softening of the $J_{\mathrm{c}}$ power law decay determined by the ratio $\alpha_{\text {nanostr. }} / \alpha_{\text {ref. }}$, and (2) the decrease of the self-field $J_{\mathrm{c}}$ given by $J_{\mathrm{c} \text { nanost. }}^{\mathrm{sf}} / J_{\mathrm{c} \text { ref. }}^{\mathrm{sf}}$. While (2) depends on the reduction of cross section perpendicular to the current flow, $S_{\text {red }}$ (Figure 6.12a), the $\alpha$ value can be correlated with the length of milled nanowalls projected along the current direction (perpendicular to the pinning force), $x_{\text {nanowall }}$. Figure 6.13a illustrates the linear dependence of $\alpha_{\text {nanostr. }} / \alpha_{\text {ref. }}$ with $x_{\text {nanowall }}$, clearly evidencing that nanowalls are the main parameter controlling the observed $\alpha$ reduction. The improvement in the pinning performance due to the presence of nanowalls can be better observed in Figure 6.13b, where the variation of the maximum pinning force, due to the 

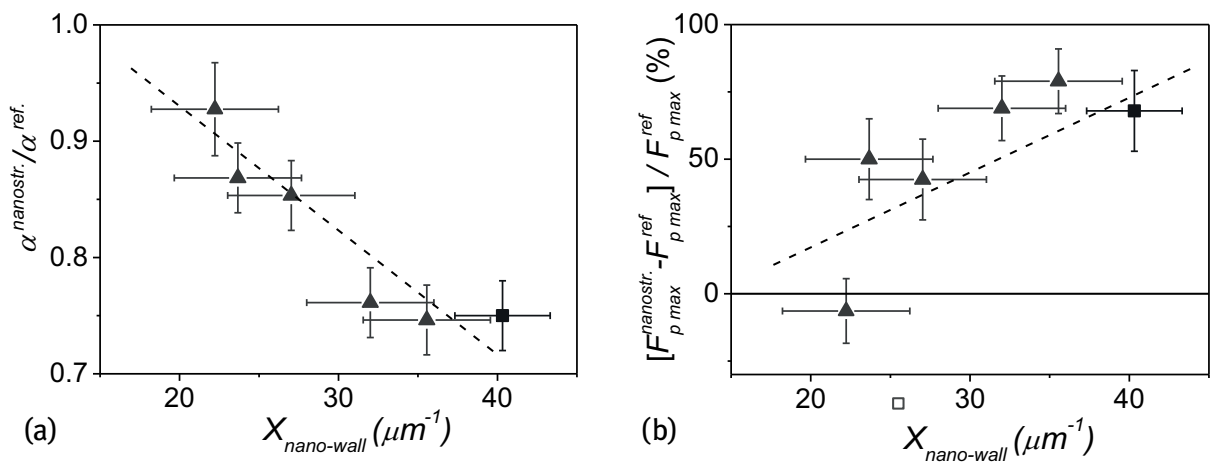

Fig. 6.13: (a) Ratio of $\alpha$ parameter and (b) variation of the maximum pinning force at $77 \mathrm{~K}$ versus $x_{\text {nanowall }}$ (defined in the text) for different nanostructured bridges. Triangular and square symbols correspond to samples with blind triangular antidots and linear trenches, respectively. Dashed lines are a guide for the eyes.

patterning, is plotted versus $x_{\text {nanowall }}$. A positive pinning force enhancement, as large as $70 \%-80 \%$, is obtained if (1) and (2) are compensated.

Further evidence that nanowalls are acting as very effective pinning sites comes from Bitter decoration experiments. Figures 6.14 and 6.15 show Bitter decoration images of two different systems with blind nanostructures.

In the first case, a reference bridge and a bridge half-covered with blind trenches, fabricated by FIB, decorated at $1.6 \mathrm{mT}$, are shown. While no ordering at all is obtained in the reference bridge, a clear periodicity on the vortex position is induced by the nanofabricated trenches as observed in the longitudinal fringes appearing at the autocorrelation function. This periodicity is associated to the vortex lattice interaction with the trenches that tends to pin the vortices along them.

In the example shown in Figure 6.15, we analyze the vortex distribution in a system with a triangular blind antidot, fabricated by EBL and decorated at $3.3 \mathrm{mT}$. In order to be able to evaluate enough density of vortices along the antidot walls we have written a larger antidot than the ones patterned on studied bridges. The autocorrelation function has been evaluated in two areas near the walls and inside the antidot. The ordering of vortices along the nanowalls, observed in the autocorrelation images evidences that, as observed from the transport measurements, the main parameter controlling vortex pinning in blind milled nanostructures are the nanowalls. This nanowall pining has been associated to a reduction of the order parameter near the nanowalls by localized deoxygenation or amorphization of the YBCO structure, induced by the nanofabrication technique, acting as a barrier for vortex motion [43]. 

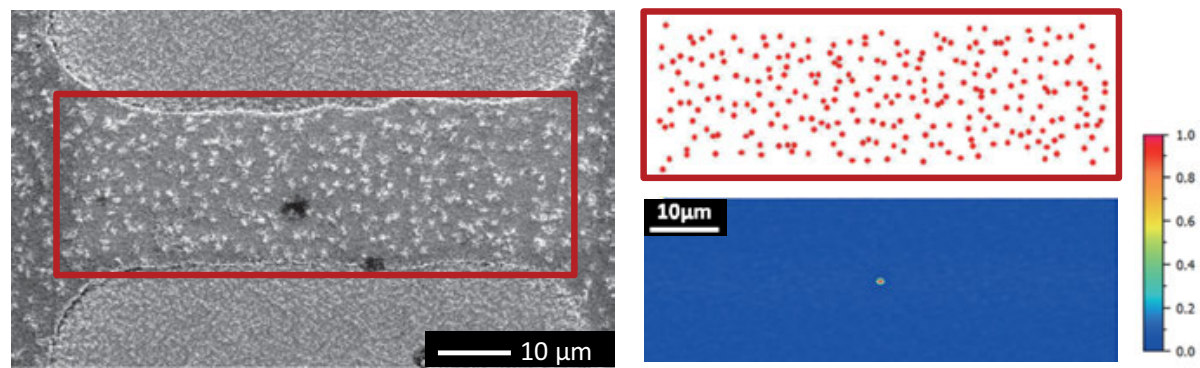

(a)
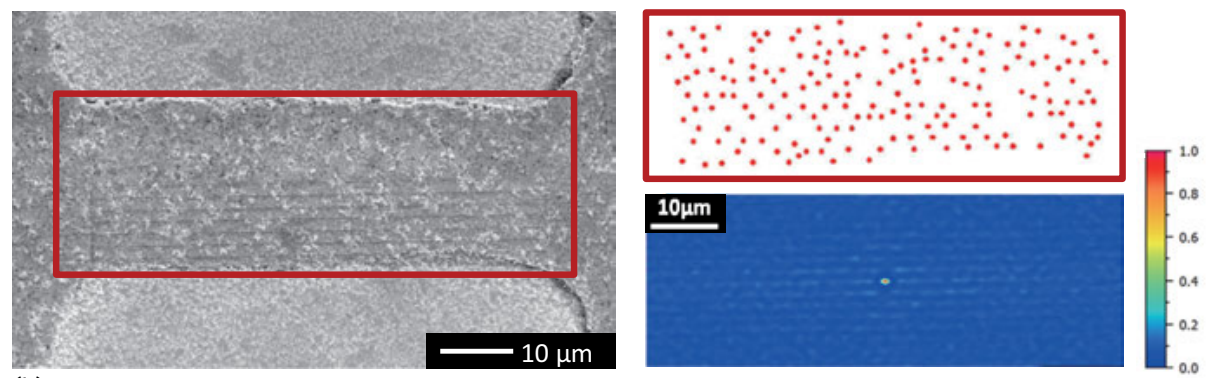

(b)

Fig. 6.14: SEM images showing the Bitter decoration at $1.6 \mathrm{mT}$ and $4.2 \mathrm{~K}$ of a CSD-YBCO (a) reference bridge and (b) bridge half-covered with blind trenches, with the associated vortex positions and autocorrelation functions.
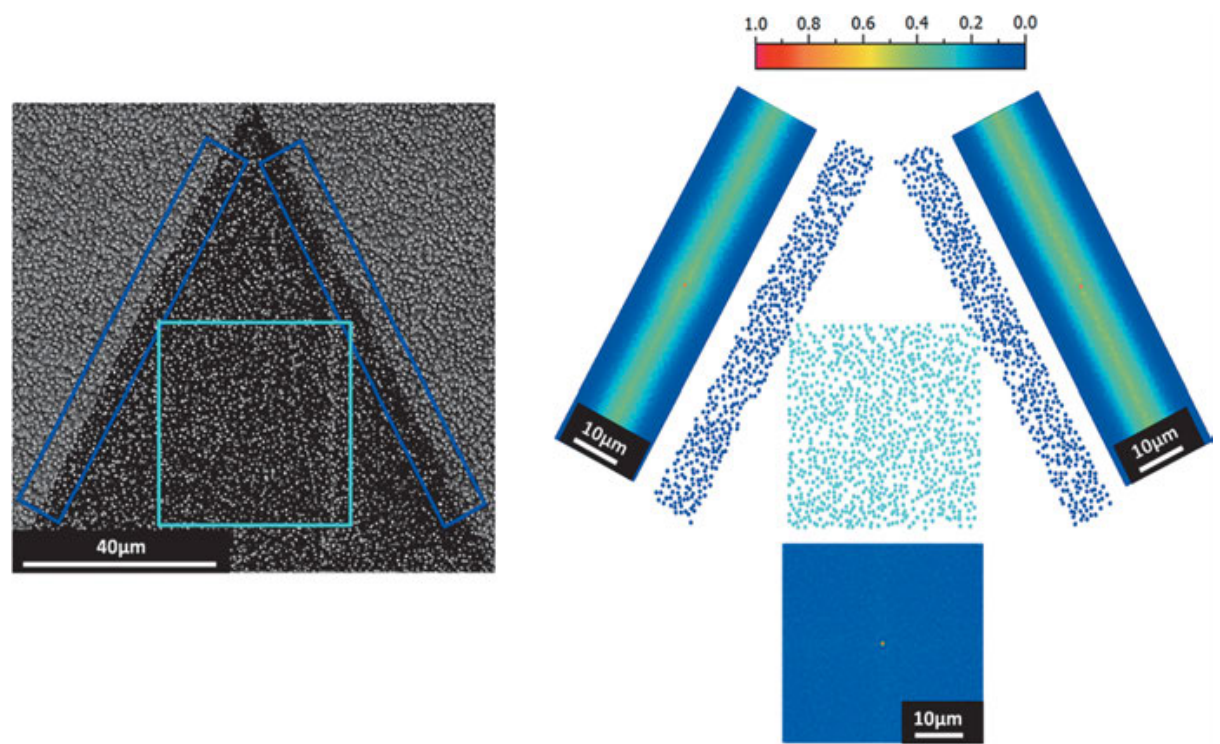

Fig. 6.15: SEM image showing the Bitter decoration at $3.3 \mathrm{mT}$ and $4.2 \mathrm{~K}$ of a CSD-YBCO film patterned with a triangular blind antidot with the vortex positions determined at different regions of the film and the associated autocorrelation functions. 


\section{Ratchets}

In the previous section we demonstrated that by means of blind antidot patterning we are able to generate artificial defects (nanowalls) acting as strong pinning sites, being stronger than intrinsic pinning. Here we will exploit this process in order to create and study ratchet pinning potentials in high-temperature superconductors. Rectified motion of particles under an asymmetric potential (ratchet effect) is important to providing deeper understanding of several microscopic ratchet systems and they are potentially useful for many novel electronic and molecular devices such as rectifiers, pumps, switches, or transistors [44, 45].

In general, ratchet systems based on superconducting materials (controlled transport of magnetic flux quanta) are focused on the study of vortex dynamics determined by the motion of a few interacting particles, since the ratchet effect disappears at high magnetic fields [46]. The use of high-temperature thin films, with a very rich $H-T$ vortex phase diagram, enables us to study systems containing many particles and their collective interaction.

Asymmetric pinning centers are generated on the bridges by patterning arrays with different size, depth, and distribution of triangular blind antidots via Focus Ion Beam (FIB) and Electron Beam Lithography (EBL). We will first explore two different artificial lattices with ordered arrays of symmetric and asymmetric blind antidots. Figure 6.16 shows SEM pictures of a $250 \mathrm{~nm}$ CSD-YBCO film patterned with lattices of triangular (asymmetric) and diamond (symmetric) antidots, with a lateral size of $0.8 \mu \mathrm{m}$, depth of $80 \mathrm{~nm}$, and spaced out $2.4 \mu \mathrm{m}$. The effect of the antidot symmetry in the vortex dynamics has been evaluated by measuring the critical current density under positive, $J_{\mathrm{c}}^{+}$and negative, $J_{\mathrm{c}}^{-}$, applied dc current, at a given magnetic field, for the two systems. By inverting the sign of the current we are inverting the sign of the driving Lorentz force $(\boldsymbol{J} \times \boldsymbol{B})$ and thus the vortex motion direction (see Figure 6.17a).

Positive and negative branches of the $J$ - $E$ curves measured for a track with triangles and diamonds, at $65 \mathrm{~K}$ and $20 \mathrm{mT}$, are plotted in Figure 6.17(b). While the curves obtained for the bridge with symmetric pinning potentials are independent of the driving force sign, a clear hard vortex moving direction, detected as a $J_{c}$ enhancement, is found in the track with asymmetric antidots. The correlation between the antidot geometry with the experimental $J-E$ curves obtained allows us to work out that dissipation is determined by motion of external vortices (located outside the antidots). At positive applied current, external vortices encounter very similar pinning potentials; tilted nanowalls in both triangular and diamond antidots, and hence very similar $J$ $E$ curves are measured. By inverting the direction of the current, external vortices in the system with triangles must flow against perpendicular nanowalls, which require a larger driving force than the one necessary to overcome the tilted edges (see Figure 6.17a).

Figure 6.17c shows the field dependence of the critical current density rectification $\Delta J_{\mathrm{c}}=\left[J_{\mathrm{c}}^{+}-J_{\mathrm{c}}^{-}\right]$obtained at $65 \mathrm{~K}$ for the track with diamond and triangular antidots. 

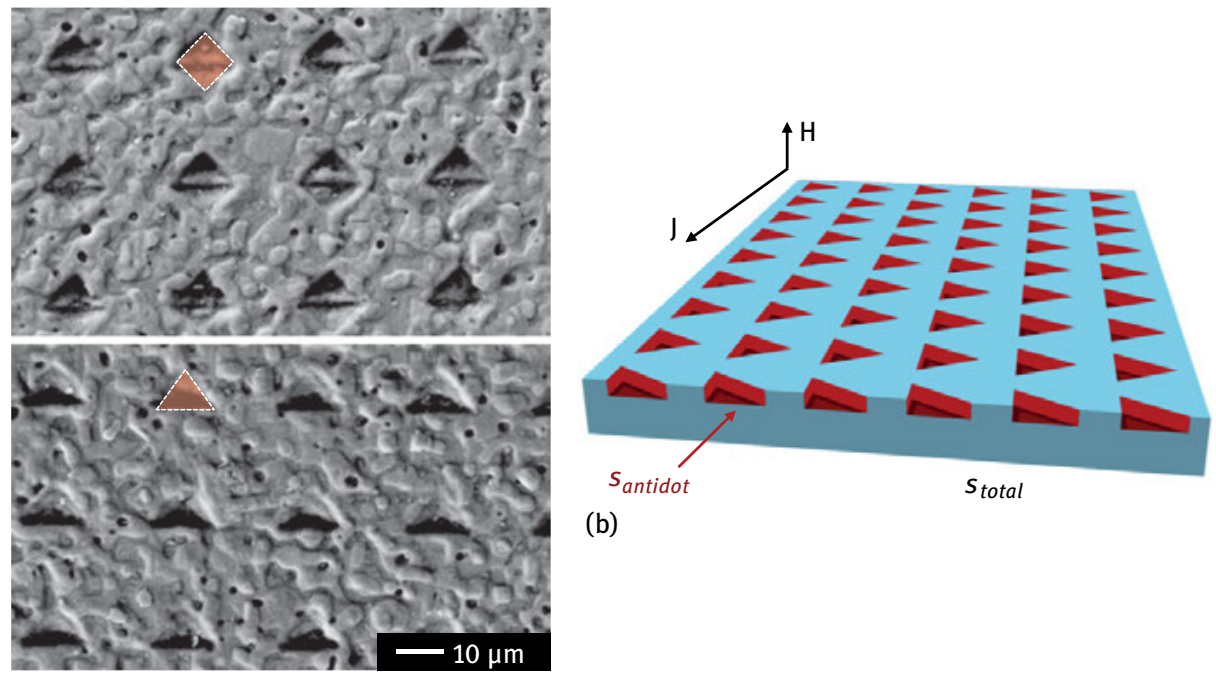

(b)

(a)

Fig. 6.16: (a) SEM images of two CSC-YBCO bridges patterned with blind diamond (top) and triangular (bottom) antidots. (b) Schematic drawing showing the cross section of bridges patterned with triangular antidots. Red area corresponds to the area occupied by blind antidots (santidot) and stotal is the total cross section of the unpatterned bridge.
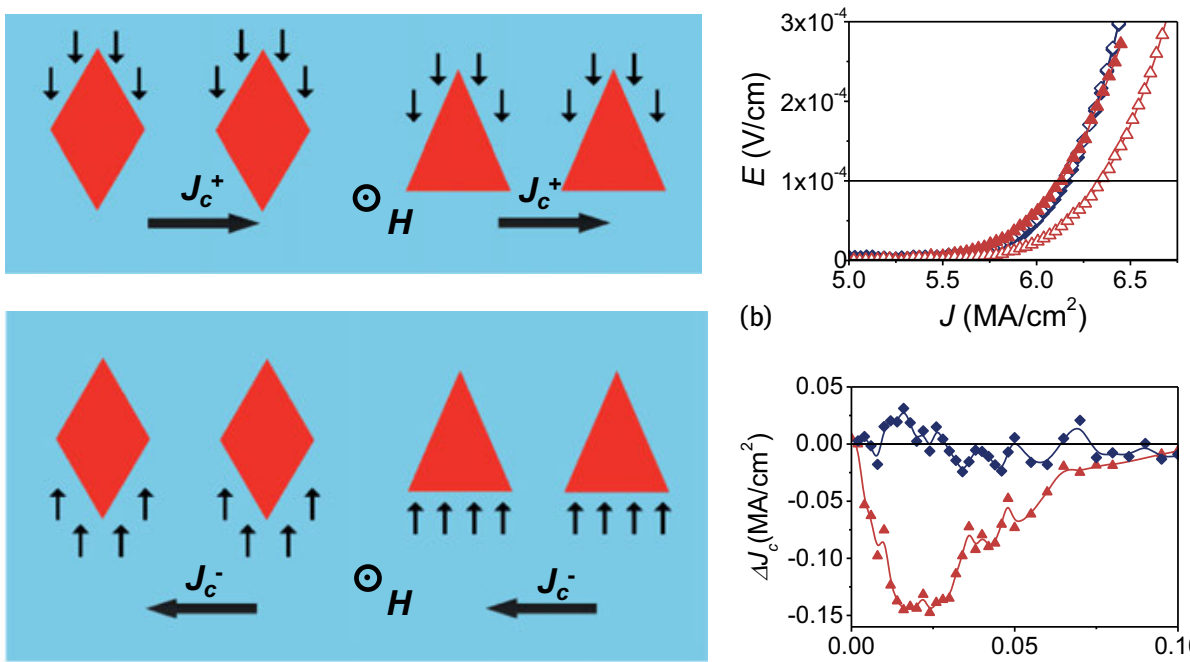

(a)

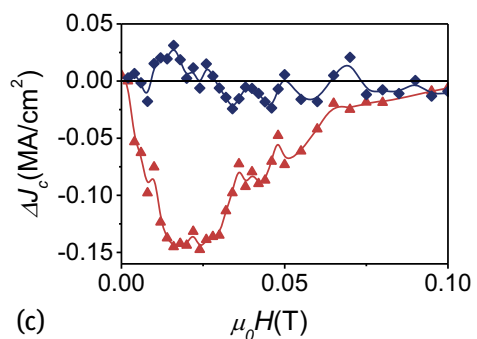

Fig. 6.17: (a) Schematic drawing of external vortex motion under positive and negative current for a sample patterned with diamond and triangular antidots. (b) Positive (closed symbols) and negative (open symbols) branches of $J-E$ curves measured for a track with triangles (triangles) and diamonds (diamonds), at $65 \mathrm{~K}$ and $20 \mathrm{mT}$. For a direct comparison $J^{+}$and $J^{-}$are shown in the same quadrant. The line shows the electric field criteria chosen to determine $J_{c}$. (c) Magnetic field dependence of the critical current density rectification $\Delta J_{c}=\left[J_{c}^{+}-J_{c}^{-}\right]$obtained at $65 \mathrm{~K}$ for a bridge with triangular (triangles) and diamond (diamonds) blind antidots. 
As observed in Figure 6.17(b) for $20 \mathrm{mT}$, no difference between $J_{\mathrm{c}}^{+}$and $J_{\mathrm{c}}^{-}$at any value of the magnetic field is found for the bridge with diamonds while a clear asymmetric response of $J_{\mathrm{c}}$ with the current direction is detected for the bridge with asymmetric pinning potential (triangles).

Figure 6.18a shows $\Delta J_{\mathrm{c}}$ obtained as a function of the magnetic field for three samples with different triangular arrays of blind antidots. In all cases the antidots were patterned $70 \mathrm{~nm}$ deep and we changed their lateral size and spacing. We plot the $\Delta J_{\mathrm{c}}$ normalized to self-field $J_{\mathrm{c}}$ such that we are able to compare net rectification effects in systems with different $J_{\mathrm{c}}$ values. The vortex ratchet effect is clearly observed in all patterned films confirming the existence of an asymmetric pinning potential induced by the presence of blind triangles. It is important to remark however, that both the polarity and the amplitude of the rectified effect strongly depend on the geometry of the patterns. While for sample \#1 the direction of the current that drives vortices along the hard moving direction is $J_{\mathrm{c}}^{-}$, as in the ratchet system shown in Figure 6.17, the opposite occurs in samples \#2 and \#3. Thus, depending on the pattern geometry ratchet systems show negative rectification with $J_{\mathrm{c}}^{-}>J_{\mathrm{c}}^{+}\left(\Delta J_{\mathrm{c}}<0\right)$ or positive rectification with $J_{\mathrm{c}}^{+}<J_{\mathrm{c}}^{-}\left(\Delta J_{\mathrm{c}}<0\right)$. Moreover, not only the polarity but also the maximum rectified critical current density, $\Delta J_{\mathrm{c}}^{\max } / J_{\mathrm{c}}^{\mathrm{sf}}$, depend on the antidot pattern.
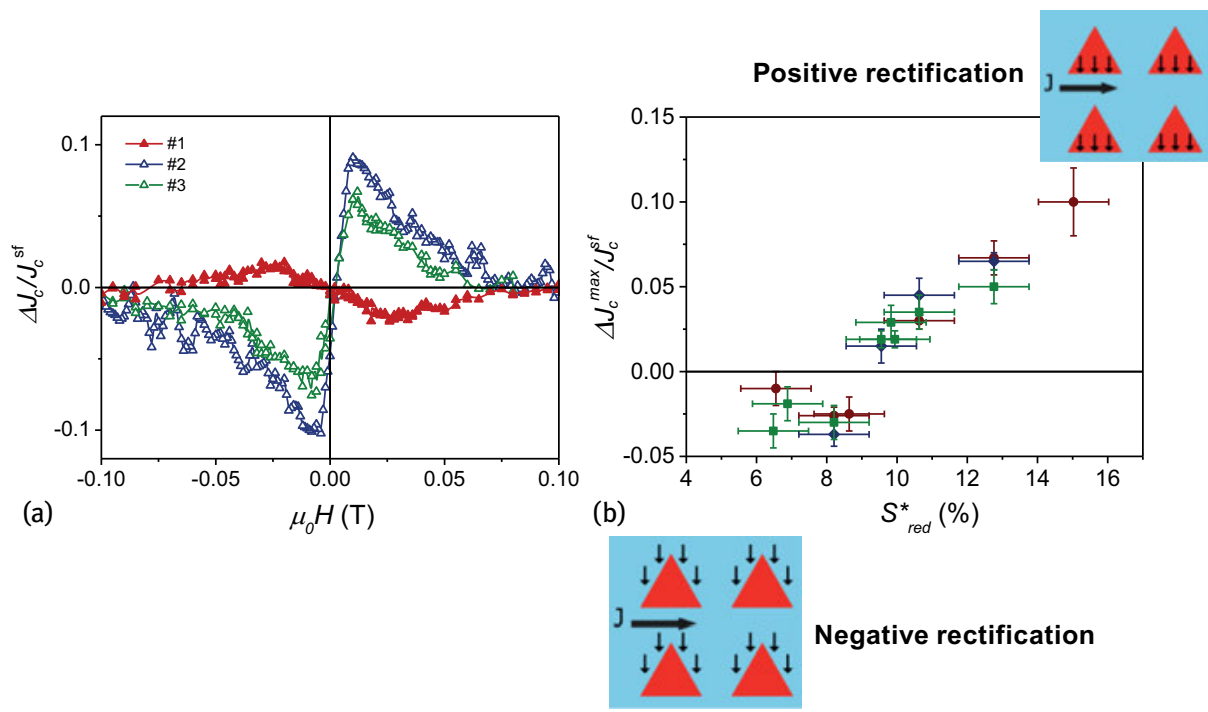

Fig. 6.18: (a) Normalized rectification of $J_{\mathrm{c}}$ at $77 \mathrm{~K}$ as a function of the magnetic field for samples with different patterned triangular antidot lattices showing positive (\#2 and \#3) and negative (\#1) rectification. (b) Maximum value normalized rectification as a function of the $S_{\text {red }}^{*}$ parameter for different systems at $77 \mathrm{~K}$ (circles), $65 \mathrm{~K}$ (squares), and $50 \mathrm{~K}$ (diamonds). Inset shows schematic drawings of vortex motion in a system with positive (top) and negative (bottom) rectification when positive current and magnetic field are applied. 
Figure $6.18 \mathrm{~b}$ displays the strength and sign of the rectified vortex motion, determined by $\Delta J_{\mathrm{c}}^{\max } / J_{\mathrm{c}}^{\mathrm{sf}}$ at positive magnetic fields, for several systems containing different arrays of triangles at various temperatures. The $\Delta J_{\mathrm{c}}^{\max } / J_{\mathrm{c}}^{\mathrm{sf}}$ values obtained can been correlated with a dimensionless parameter, $S_{\text {red }}^{*}$, that considers the maximum area reduction of the current cross section in each system and the asymmetry introduced by the triangular shape as:

$$
S_{\text {red }}^{*}(\%)=100 \frac{S_{\text {antidot }}}{S_{\text {total }}} \cos (\theta),
$$

where $s_{\text {antidot }}$ is the maximum area of blind antidots perpendicular to the current flow (red area in Figure 6.16b), stotal the cross-sectional area of the unpatterned bridge, and $\theta$ the angle between the tilted edges of the triangle and the driving force direction.

Depending on the cross section of the ratchet system, quantified by $S_{\text {red }}^{*}$, systems with negative or positive rectification can be found which is in agreement with initial dissipation from external (located outside the antidots) or internal (located within the antidots) vortices, respectively (see schematic drawings in Figure 6.18b). This picture was corroborated by numerical simulations performed in bridges with different antidot sizes which confirmed that maximum dissipative areas are located either below or outside the antidots depending on the final cross section of patterned bridges [47].

In conclusion, we have demonstrated geometrically controlled rectified vortex motion effects in YBCO films patterned with asymmetric triangular blind antidots. In these systems, both the steepness and sign of the ratchet potential can be tailored with the geometry (size, depth, and distribution) of the patterned triangles. All ratchet devices present rectified motion up to high fields allowing one to study rectification effects in different vortex dynamic regimes within the $H-T$ phase diagram. In particular, different dissipation mechanisms have been identified for ratchets with positive and negative rectification, depending on the nature of vortices initiating the dissipation [47]. Thus, using a system based on a high-temperature superconductor we provide a useful toolbox for studying transport of multiple particles at the nanoscale.

\subsection{General conclusions}

We demonstrated the potentiality of Focused Ion Beam and Electron Beam Lithography techniques to manipulate the vortex pinning landscape of high-quality CSDYBCO thin films. CSD appears to be a low-cost, versatile, and scalable growth process for the preparation of high critical current YBCO films with a high density of intrinsic pinning sites. We used efficient high-resolution lithography tools to tailor the particular intrinsic microstructure of CSD-YBCO films, generating several model systems for vortex pinning studies. Full and blind antidots with different geometry have been patterned in several YBCO bridges, allowing one to study different physical phenomena in high-temperature superconducting films, which show a very rich $H$ - $T$ vortex 
phase diagram. The interaction of the patterned structures with vortices have been explored within the single vortex pinning and collective regimes by using transport measurements and Bitter decoration images. Unique and interesting effects, such us artificial granularity, nanowall pinning, and rectification effects have been generated and deeply revised in this chapter.

Acknowledgment: This work has been supported by MINECO (MAT2014-51778-C21R, MAT2011-28874-C02-01, Programa CONSOLIDER, CSD 2007-00041), Generalitat de Catalunya (2014-SGR-00753), European Union, FP7- EU-NMP-LA-2012-280432 EUROTAPES project and Cost Action MP1201. ICMAB acknowledges the Severo Ochoa Center of Excellence mention from MINECO.

\section{Bibliography}

[1] Malozemoff AP, Mannhart J, Scalapino D. Phys. Today 58:41-47, 2005.

[2] Malozemoff AP, Second-Generation High-Temperature Superconductor Wires for the Electric Power Grid, in: Clarke DR. (Ed.) Annu. Rev. Mater. Res., Vol. 42:373-397, 2012.

[3] Ahn CH, Triscone JM, Mannhart J. Nature 424:1015-1018, 2003.

[4] Rupich MW, Li XP, Thieme C, Sathyamurthy S, Fleshler S, Tucker D, Thompson E, Schreiber J, Lynch J, Buczek D, DeMoranville K, Inch J, Cedrone P, Slack J. Supercond. Sci. Tech. 23:014015, 2010.

[5] Shiohara Y, Taneda T, Yoshizumi M. Jpn. J. Appl. Phys. 51:010007, 2012.

[6] Obradors X, Puig T. Supercond. Sci. Tech. 27:044003, 2014.

[7] Maiorov B, Baily SA, Zhou H, Ugurlu O, Kennison JA, Dowden PC, Holesinger TG, Foltyn SR, Civale L. Nature Materials 8:398-404, 2009.

[8] Gutierrez J, Llordes A, Gazquez J, Gibert M, Roma N, Ricart S, Pomar A, Sandiumenge F, Mestres N, Puig T, Obradors X. Nature Materials 6:367-373, 2007.

[9] Macmanus-Driscoll JL, Foltyn SR, Jia QX, Wang H, Serquis A, Civale L, Maiorov B, Hawley ME, Maley MP, Peterson DE. Nature Materials 3:439-443, 2004.

[10] Llordes A, Palau A, Gazquez J, Coll M, Vlad R, Pomar A, Arbiol J, Guzman R, Ye S, Rouco V, Sandiumenge F, Ricart S, Puig T, Varela M, Chateigner D, Vanacken J, Gutierrez J, Moshchalkov V, Deutscher G, Magen C, Obradors X. Nature Materials 11:329-336, 2012.

[11] Shiohara Y, Yoshizumi M, Takagi Y, Izumi T. Physica C 484:1-5, 2013.

[12] Yamada Y, Takahashi K, Kobayashi H, Konishi M, Watanabe T, Ibi A, Muroga T, Miyata S, Kato T, Hirayama T, Shiohara Y. Appl. Phys. Lett. 87:132502, 2005.

[13] Kang S, Goyal A, Li J, Gapud AA, Martin PM, Heatherly L, Thompson JR, Christen DK, List FA, Paranthaman M, Lee DF. Science 311:1911-1914, 2006.

[14] Xu A, Delgado L, Khatri N, Liu Y, Selvamanickam V, Abraimov D, Jaroszynski J, Kametani F, Larbalestier DC. Apl Materials 2:046111, 2014.

[15] Schwartz RW, Schneller T, Waser R. Comptes Rendus Chimie 7:433-461, 2004.

[16] Bassiri-Gharb N, Bastani Y, Bernal A. Chem. Soc. Rev. 43:2125-2140, 2014.

[17] Obradors X, Puig T, Gibert M, Queralto A, Zabaleta J, Mestres N. Chem. Soc. Rev. 43:22002225, 2014.

[18] Obradors X, Puig T, Ricart S, Coll M, Gazquez J, Palau A, Granados X. Supercond. Sci. Tech. 25:123001, 2012. 
[19] Araki T, Hirabayashi I. Supercond. Sci. Tech. 16:,R71-R94, 2003.

[20] Obradors X, Puig T, Pomar A, Sandiumenge F, Pinol S, Mestres N, Castano O, Coll M, Cavallaro A, Palau A, Gazquez J, Gonzalez JC, Gutierrez J, Roma N, Ricart S, Moreto JM, Rossell MD, van Tendeloo G. Supercond. Sci. Tech. 17:1055-1064, 2004.

[21] Obradors X, Puig T, Pomar A, Sandiumenge F, Mestres N, Coll M, Cavallaro A, Roma N, Gazquez J, Gonzalez JC, Castano O, Gutierrez J, Palau A, Zalamova K, Morlens S, Hassini A, Gibert M, Ricart S, Moreto JM, Pinol S, Isfort D, Bock J. Supercond. Sci. Tech. 19:S13-S26, 2006.

[22] Obradors X, Martinez-Julian F, Zalamova K, Vlad VR, Pomar A, Palau A, Llordes A, Chen H, Coll M, Ricart S, Mestres N, Granados X, Puig T, Rikel M. Physica C 482:58-67, 2012.

[23] Farjas J, Camps J, Roura P, Ricart S, Puig T, Obradors X. Thermochim. Acta 544:77-83, 2012.

[24] Palmer X, Pop C, Eloussifi H, Villarejo B, Roura P, Farjas J, Calleja A, Palau A, Obradors X, Puig T, Ricart S. Supercond. Sci. Tech. 29:024002, 2016.

[25] Van Driessche I, Feys J, Hopkins SC, Lommens P, Granados X, Glowacki BA, Ricart S, Holzapfel B, Vilardell M, Kirchner A, Baecker M. Supercond. Sci. Tech. 25:065017, 2012.

[26] Vilardell M, Granados X, Ricart S, Van Driessche I, Palau A, Puig T, Obradors X. Thin Solid Films 548:489-497, 2013.

[27] Kimura K, Hironaga R, Nakamura T, Takahashi Y, Koizumi T, Hasegawa T, Higashikawa K, Inoue M, Kiss T, Yoshizumi M, Izumi T, Shiohara Y. IEEE T. Appl. Supercon. 25:6604204, 2015.

[28] Gazquez J, Coll M, Roma N, Sandiumenge F, Puig T, Obradors X. Supercond. Sci. Tech. 25:065009, 2012.

[29] Rouco V, Palau A, Guzman R, Gazquez J, Coll M, Obradors X, Puig T. Supercond. Sci. Tech. 27:125009, 2014.

[30] Coll M, Ye S, Rouco V, Palau A, Guzman R, Gazquez ], Arbiol J, Suo H, Puig T, Obradors X. Supercond. Sci. Tech. 26:015001, 2013.

[31] Cayado P, De Keukeleere K, Garzon A, Perez-Mirabet L, Meledin A, De Roo J, Valles F, Mundet B, Rijckaert H, Pollefeyt G, Coll M, Ricart S, Palau A, Gazquez J, Ros J, Van Tendeloo G, Van Driessche I, Puig T, Obradors X. Supercond. Sci. Tech. 28:124007, 2015.

[32] Rouco V. PhD. Thesis, http://hdl.handle.net/10803/133329, 2014.

[33] Palau A, Bartolome E, Llordes A, Puig T, Obradors X. Supercond. Sci. Tech. 24:125010, 2011.

[34] Zuev YL, Christen DK, Wee SH, Goyal A, Cook SW. Appl. Phys. Lett. 93:172512, 2008.

[35] Fasano Y, Menghini M. Supercond. Sci. Tech. 21:023001, 2008.

[36] Menghini M, Kramer RBG, Silhanek AV, Sautner J, Metlushko V, De Keyser K, Fritzsche J, Verellen N, Moshchalkov VV. Phys. Rev. B 79:144501, 2009.

[37] Luccas RF. PhD. Thesis, http://hdl.handle.net/10803/294271, 2011.

[38] Luccas RF, Granados X, Obradors X, Puig T. Physica C 505:47-54, 2014.

[39] Palau A, Puig T, Obradors X, Jooss C. Phys. Rev. B 75:054517, 2007.

[40] Palau A, Puig T, Obradors X, Pardo E, Navau C, Sanchez A, Usoskin A, Freyhardt HC, Fernandez L, Holzapfel B, Feenstra R. Appl. Phys. Lett. 84:230-232, 2004.

[41] Bartolome E, Palau A, Gutierrez J, Granados X, Pomar A, Puig T, Obradors X, Cambel V, Soltys J, Gregusova D, Chen DX, Sanchez A. Phys. Rev. B 76:094508, 2007.

[42] Rouco $V$ et al. (To be published).

[43] Palau A, Rouco V, Luccas RF, Obradors X, Puig T. Physica C 506:178-183, 2014.

[44] Howard J. Nature 389:561-567, 1997.

[45] Astumian RD, Hanggi P. Phys. Today 55:33-39, 2002.

[46] Plourde BLT. IEEE T. Appl Supercon. 19:3698-3714, 2009.

[47] Rouco V, Palau A, Monton C, Del-Valle N, Navau C, Sanchez A, Obradors X, Puig T. New J. Phys. 17:073022, 2015. 
Brought to you by | CSIC - Unidad Coordinacion Bibliotecas 